\title{
Research on Remote Sensing and Geographical Information System and Exploration for Its Practical Application in Land Utilization Management
}

\author{
Shiqiang Li \\ Remote Sensing and GIS Research Institute, Chengdu University of Technology, \\ Chengdu, 610059, China \\ Faculty of Geography and Resource Science, Sichuan Normal University, Chengdu, \\ 610066, China
}

\begin{abstract}
With continuous development of China's social economy in recent years, it also becomes more and more complicated for the form of land utilization. At the same time, severe damages were caused to natural environment and land resources. With continuous changes in population and environment, some unreasonable issues are inevitable in the exploitation and utilization process of land resources. For this circumstance, the author in this paper made a further research on the practical application of remote sensing and geographical information system in land utilization management, and made an in-depth discussion on practical application of RS and GPS in land utilization management from such perspectives as the connotation of remote sensing \& geographical information system, the combination of remote sensing technology with geographical information system and the application of RS and GIS in land utilization management, with expectations to provide some reference to relevant researches on land utilization management.
\end{abstract}

Key words: remote sensing technology, geographical information system, land utilization management land utilization management

With continuous development of the scientific and technological level in recent years, it becomes more and more complicated for land utilization management. The rate of population increase becomes faster and faster. In the development process of land resources, some issues are inevitable. Under such circumstance, RS and GIS are of dynamic features in the development process. In the monitoring process of dynamic land changes, remote sensing, geographical information system and others provided more high-tech means for the completion of these works. RS and GIS are of obvious technical advantages. Better results will be obtained, if RS and GIS can 
be properly combined in practical works. For this idea, the combination of RS and GIS was practically applied in the process of the second land survey. The application of remote sensing and geographical information system brought forth fascinating benefits.

\section{Connotation of remote sensing \& geographical information system}

\subsection{Analysis on remote sensing}

Co-called remote sensing refers to remote perception of an object. In other words, an object can be perceived from a long distance with the assistance of remote sensing under the precondition of no direct contact with the object. From a narrow sense, remote sensing is to obtain the surface information in high altitude or from long distance through the application of camera or other sensors and then further realize the understanding of size and other mutual relations of objects on the ground through data processing and other links. In the use process, remote sensing technology mainly includes active remote sensing and passive remote sensing. The former presents radar and use of manual electromagnetic radiant point. The latter one presents camera and others.

In the use process, remote sensing technology is featured by wide field of vision, much information, fast speed, less restraints and extensive application. The application of remote sensing technology provides new detective means for survey on natural resources and monitoring and forecasting of global information resources and oceanic atmosphere. At present, this technology has been widely applied into the production of national economy. At the same time, it also glows strong vitality. Remote sensing technology is a multi-platform and multi-level three-dimensional observation system, mainly including means of delivery, information pre-processing and other contents. Surface receiving station is mainly responsible for receiving and processing of remote sensing information and finishing the information through relevant devices. Computer data processing and other means are used to analyze and use useful information in remote sensing data. In the future, remote sensing technology will be provided with higher and higher resolution ratio. Moreover, its range of wave band will also be expanded from simplicity gradually to multi-polarization. The research is made from various perspectives, such as qualitative photography and quantitative analysis.

\subsection{Analysis on geographical information system}

RS mainly refers to analysis on geographic space data under the support of 
computer software and hardware, provision of various kinds of geographic information and application such information into the computer system. As a high-tech discipline, geographical information system includes geography, computer science, environmental sciences and other sciences. At the same time, geographical information system also belongs to a kind of interdisciplinary subject. Computer science is the core of this subject. Spatial analysis is the basic technique of this subject. From the perspective of content, RS can be divided into comprehensive and special geographical information systems. In terms of software feature, RS can be divided into tool-type and practical geographical information systems. From the perspective of system function, RS can be divided into topographic information system, population information system and several other types. In terms of data structure, RS can be divided into vector type, grating type, mixed type, object-oriented type and others. With respect to software composition, RS can be divided into component type, network type and other types of geographical information system.

Basic functions of GIS are data collection, map editing and others. Managed data mainly includes geographic data on two-dimensional or three-dimensional space. In the process of managing the data, GIS mainly proceeds through the form of layer, merging data in different types into a layer. This management mode is very convenient in practical application. At present, GIS has been widely emphasized. There are more and more related software. A majority of GIS software manufacturers made continuous innovations to the function of GIS and fully absorbed the function of GIS. To deepen users' understanding of different software, related organizations in some countries also tested software. At present, China has already tested domestic software for many times. Viewed from the current situation, geographical information system will manage spatial data structure and obtain and integrate special data in the future. At the same time, it trends to be intelligent, so as to promote sharing of GIS data.

\section{Combination of remote sensing technology with geographical information system}

2.1 RS is important information source of GIS.

In terms of obtaining surface information, remote sensing technology is a brand new technological means, with which relevant remote sensing data can be obtained through aerospace. Moreover, resources in pathless regions can be also further 
detected. This technology can be used to obtain information which can not be obtained through conventional means. Remote sensing technology can be used for dynamic monitoring of various resources and environmental data. Just because of this, remote sensing information becomes a very important information source of geographical information system. The time for information processing is shortened to a certain range, winning certain time for forecasting.

\subsection{Action of GIS toward RS}

Remote sensing has huge amount of data. At present, it is very difficult for existing remote sensing data processing to meet practical needs. How to effectively use and manage remote sensing becomes a key issue. This issue is mainly decided by whether it can be effectively controlled through geographic information. Whether we are able to develop such system becomes a key factor for the solution to this issue. Geographical information system can receive different spatial data. Viewed from this point, spatial data can be analyzed and processed by inputting remote sensing data into geographical information system. Moreover, the function of remote sensing data can be maximized.

\subsection{Promotion of GIS for RS drawing}

The combination of GIS and RS went through a long development course. In the combination process, the two items need to process remote sensing photos into special manuscript bases, input into geographical information system and then output special information through computer. Under the support of GIS, special data can be automatically provided by remote sensing image. Further realization is made to the image processing function of RS by utilizing remote sensing image. On this basis, the image is edited and compiled accordingly, so as to maximize various drawing elements, provide effective information guarantee to special drawing in a better way, edit special images by combining special needs and provide a great deal of information images.

\subsection{Combination of GIS with RS image processing}

The combination of GIS and RS image processing can develop interchange through many approaches, for instance, data port. Interchange can be realized between independent geographical information system and image analysis; or information sharing can be realized through image processing software after inputting processed results into RS. Additionally, a complete system can be formed jointly by image processing system and geographical information system. Thus, the image 
processing system will become a complete integrated system. In this system, remote sensing and geographical information system are included at the same time. In the layer structure, this system can coordinate grating and vector data and generate a comprehensive model in real life. At the same time, this model is used for determination by combining relevant spatial expression method and other functions. GIS and RS are independent from each other. However, the two items are of close relations. Under the support of RS, GIS information is of present situation feature. At the same time, relevant auxiliary data in GIS also provides conditions for the improvement to classification accuracy of RS. For GIS, RS is served as a major approach for data updating. Comprehensive development and utilization of RS also obtained further supports from GIS.

\section{Application of RS and GIS in land utilization management}

\subsection{Application of RS and GIS in land dynamic monitoring}

One the one hand, reasonable utilization of land resources is one of basic state policies in China. Whether land resources are reasonably utilized is directly correlated with political stability and social economy in China, especially in the ecological system. Land utilization is a sensitive factor, and it will be dually influenced by natural factors and social factors. On this basis, land utilization plays an important role in sustainable research. Traditional survey approaches are still used at present. Currently, traditional survey mode was used for the second land use survey in Chinese organizations at the county level. This survey mode is mainly reflected in the following way: understand changes in land utilization by data reported by related organizations. Traditional measuring tools are main means. Manual measurement is served as the main survey method. This method has many issues. It has not high efficiency. At the same time, it fails to obtain land utilization variation information with strong present situation feature. Therefore, it fails to reflect timely and accurately reflect land utilization changes in spatial distribution or to analyze or evaluate such changes in land utilization. The existence of these factors results in certain resistance to other works. For instance, great difficulties are caused for current situation evaluation of regional sustainable development. Therefore, it is of great importance for further research and utilization of GIS, RS and other high-tech means. Dynamic changes in land utilization can be timely and accurately obtained and managed by establishing a land utilization dynamic monitoring system, so as to perform scientific management of spatial data on land 
utilization and provide effective management work to land management departments in a better way. This is of great significance for regional sustainable development. With the wide application of remote sensing technology in recent years, researches related to land utilization also become popular gradually. There are more and more researches in this aspect.

On the other hand, China has already made in-depth researches on the application of GIS and RS in dynamic monitoring of land utilization. At present, China has already accelerated its step in the research on relevant subjects of remote sensing. For the application of geographical information system in the remote sensing survey, sensing image is classified mainly through its special data. GIS has strong spatial analysis function. Through the utilization of various spatial analysis technologies and processing of original data in the database, GIS can enable users to get the latest data set and take the data set as the basis for spatial decision making. In recent years, monitoring of changes in land utilization by GIS and RS can be used to correctly reflect the trend of researches on regional changes. Upon analysis and researches by many scholars, man-machine interactive method can be used now to interpret remote sensing images. Moreover, geographical information system can be used for dynamic monitoring of land. Undoubtedly, this is an effective method for the combination of GIS and RS. In practical application, the combination of GIS and RS gives play to many advantages, such as short period, less costs and high accuracy. It is a beneficial attempt to serve sectors of national economy through high technology.

\subsection{Application of RS and GIS in survey on current situation of land utilization}

The second population census provided application space for the application of GIS and RS technology. Existing aerial photography, satellite remote sensing and other materials should be fully utilized. Moreover, present situation of land use should be surveyed according to local conditions by utilizing geographical information system and other new technologies, so as to make comprehensive surveys on current situation of land use, timely update basic drawings, make statistics of land data, and timely update the management information system. On this basis, effective improvement should be made to the modernization and informationalized level of business management of territorial resources.

\section{Conclusion:}

Based on some unreasonable issues in the exploitation and utilization process of 
land resources and insufficient research on this aspect at present, the author in this paper made a further research and analysis on practical application of RS and GPS in land utilization management from such perspectives as the connotation of remote sensing \& geographical information system, the combination of remote sensing technology with geographical information system and the application of RS and GIS in land utilization management, with expectations to provide some reference to related researches through the research in this paper.

\section{References:}

[1] Li Xuejun, Wei Ruijuan and Qu Haitao, Remote Sensing and Geographical Information System and Its Application in Land Utilization Management [J], Geomatics \& Spatial Information Technology, 2010, (2);

[2] Xu Xinhui and Xu Yanhong, Construction of Township Level Land Utilization Information System based on RS/GIS - with Fadou Township of Xichou County as an Example [J], Chinese Journal of Population Resources and Environment, 2010, (1);

[3] Meng Qingjian, Cao Weibin, Li Jiangquan, Liu Jiaodi and Xu Xipan, Design and Realization of Legion Agricultural Land Utilization Management Information System based on GIS [J], Jiangsu Agricultural Sciences, 2011, (1);

[4] Du Jiahong and Liu Xin, Application of Remote Sensing and Geographical Information System in Land Utilization Dynamic Monitoring [J], Geomatics \& Spatial Information Technology, 2011, (3);

[5] Zhao Yupeng and Han Ying, Discussion on Application of Geographical Information System in Land Management [J], Technology Innovation and Application, 2013, (36);

[6] Deng Hongyu, Qin Yanbin and Fu Yeping, Research on Design of Land Utilization Management Information System [J], Geomatics \& Spatial Information Technology, 2013, (10);

[7] Jiang Zhongcheng, Guo Dan, Dou Sen, Yu Shasha, Zhang Yuguang and Zhang Dajun, Application of Geographical Information System in Land Management [J], Jilin Agriculture, 2012, (8);

[8]. Introduction to 2007 Silver Award Engineering of Geographical Information System, Survey on Present Situation of Land Use in Heilongjiang Province 1:10000 Orthophotoquad Making [J], Geomatics World, 2008, (3). 